\title{
Flow disturbances and the development of endocardial fibroelastosis
}

Viktoria Weixler, MD, ${ }^{a}$ Gerald R. Marx, MD, ${ }^{b}$ Peter E. Hammer, PhD, ${ }^{a}$ Sitaram M. Emani, MD, Pedro J. del Nido, MD, ${ }^{a}$ and Ingeborg Friehs, MD $^{\mathrm{a}}$

\section{ABSTRACT}

Objectives: Endothelial-to-mesenchymal transition (EndMT) has been identified as the underlying mechanism of endocardial fibroelastosis (EFE) formation. The purpose of this study was to determine whether hemodynamic alterations due to valvar defects promote EndMT and whether age-specific structural changes affect ventricular diastolic compliance despite extensive surgical resection of EFE tissue.

Material and Methods: We analyzed EFE tissue from 24 patients with hypoplastic left heart syndrome (HLHS) who underwent left ventricular (LV) rehabilitation surgery at Boston Children's Hospital between December 2011 and March 2018. Six patients with flow disturbances across the aortic valve and/or mitral valve but no HLHS diagnosis and macroscopic appearance of "EFE-like tissue" in the LV were included for comparison. All samples were examined for amount of collagen/elastin production and degradation, and presence of active EndMT by histologic analysis.

Results: EFE tissue from patients with and without HLHS consisted predominantly of elastin and collagen fibers. There was no alteration in degradation activity for collagen or elastin as shown by in situ zymography. Active EndMT was found in all patients with and without HLHS with flow disturbances ("EFE-like"). In patients with HLHS, EFE infiltrated into the underlying myocardium with increasing age.

Conclusions: Patients with and without HLHS with flow disturbances due to stenotic or incompetent valves develop EndMT-derived fibrotic tissue covering the LV. When EFE recurs, it is directly associated with flow disturbances and switches to an infiltrative growth pattern with increasing age, leading to increased diastolic stiffness of the LV. (J Thorac Cardiovasc Surg 2020;159:637-46)

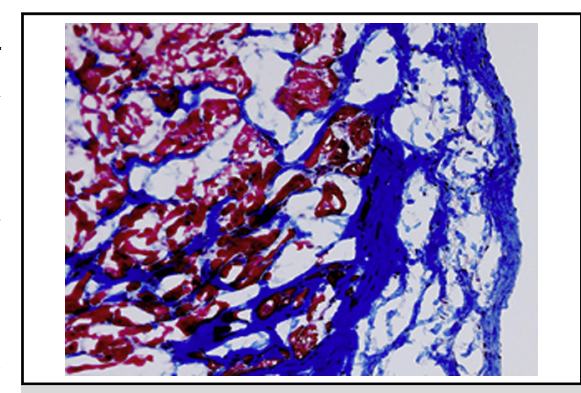

Endocardial fibroelastosis (b/ue) shows infiltrative growth pattern into the underlying myocardium (red).

\section{Central Message}

EFE shows infiltrative growth with increasing age, triggered by flow disturbances promoting EndMT as the underlying mechanism that is independent of HLHS.

\section{Perspective}

Flow disturbances exposing endocardial endothelial cells to altered shear forces are the main trigger for induction of EndMT leading to reoccurrence of EFE displaying a more infiltrative growth pattern into the underlying myocardium. These alterations are not limited to HLHS but are related to severe valvar defects with jets onto the endocardial surface.

See Commentaries on pages 647 and 649.
Endocardial fibroelastosis (EFE) was first described by Weinberg and Himmelfarb in 1943 as a thick subendocardial layer of connective tissue and elastin, encapsulating the underlying myocardium of left atrium and left ventricle (LV). ${ }^{1}$ Since then, the presence of EFE has been reported in several other cardiac diseases such

\footnotetext{
From the Departments of a Cardiac Surgery and 'bardiology, Boston Children's Hospital, Harvard Medical School, Boston, Mass.

Received for publication May 10, 2019; revisions received Aug 21, 2019; accepted for publication Aug 24, 2019; available ahead of print Oct 23, 2019.

Address for reprints: Ingeborg Friehs, MD, Department of Cardiac Surgery, Boston Children's Hospital, Harvard Medical School, 300 Longwood Ave, Boston, MA 02115 (E-mail: ingeborg.friehs@childrens.harvard.edu). $0022-5223 / \$ 36.00$

Copyright (C) 2019 by The American Association for Thoracic Surgery https://doi.org/10.1016/j.jtcvs.2019.08.101
}

as cardiomyopathies, ${ }^{2}$ infectious diseases, ${ }^{3}$ immunologic diseases, $^{4}$ and congenital heart diseases such as hypoplastic left heart syndrome (HLHS) ${ }^{5,6}$ Macroscopic and microscopic appearance led to this uniform diagnosis, but the underlying root cause of EFE development has not been examined until recently.

With surgical resection of EFE during routine rehabilitation surgery aimed at preserving the growth

- Scanning this $\mathrm{QR}$ code will take you to the article title page to access supplementary information.

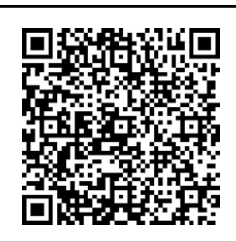




\section{Abbreviations and Acronyms \\ $\alpha$-SMA $=\alpha$-smooth muscle actin \\ $\mathrm{CD} 31=$ cluster of differentiation 31 \\ $\mathrm{EFE}=$ endocardial fibroelastosis \\ EndMT $=$ endothelial-to-mesenchymal transition \\ HLHS = hypoplastic left heart syndrome \\ $\mathrm{IQR}=$ interquartile range \\ $\mathrm{LV} \quad=$ left ventricle \\ MMP = matrix metalloproteinase \\ MRI = magnetic resonance imaging \\ MT = Masson's trichrome}

pathologic conditions such as myocardial fibrosis. ${ }^{9}$ Stimuli for EndMT include hypoxia/ischemia, ${ }^{10}$ inflammation, ${ }^{11}$ and mechanical alterations, ${ }^{12,13}$ whereof the latter plays an important role in congenital cardiac defects such as HLHS. Our focus is on EFE associated with HLHS, which encompasses a spectrum of complex congenital heart lesions with various degrees of underdevelopment of the LV and aortic/mitral valve stenosis/atresia. ${ }^{5}$ Our patient cohort included only patients with patent but stenotic mitral valves potentially suitable for LV recruitment surgery.

In up to $70 \%$ of all patients with HLHS, EFE restricts the left ventricular outflow tract, ${ }^{14}$ and surgical removal of EFE tissue has been found to result in catch-up growth of LV structures. $^{7}$ At times, several EFE resections during consecutive surgical interventions in the same patients are necessary to potentially alleviate the restrictive forces of this stiff tissue. Despite the increase in LV volume indicative of ventricular growth, the restrictive component on the LV is not resolved as the patients grow older, since they continue to present with persistently elevated end-diastolic pressures. ${ }^{15}$

We recently presented a unique case of EFE reoccurrence in a patient with HLHS with severe flow disturbances across the mitral valve, which was resolved by repetitive EFE resections and replacement of the mitral valve to normalize blood flow in the LV. ${ }^{16}$ For the first time, this report associates mechanical forces such as flow disturbances with EFE development and identifies EndMT as underlying mechanism. The purpose of this study was to determine whether hemodynamic alterations due to valvar stenosis cushions and valves, ${ }^{8}$ and it has also been described under

nted

TABLE 1. Detailed information of patients with HLHS who were consented

\begin{tabular}{|c|c|c|c|c|c|}
\hline Patient & Age & Diagnosis & Performed procedures & No. EFE resections & Localization of EFE resection \\
\hline 1 & $5 y$ & $\begin{array}{l}\text { HLHS, AS, MS } \\
\quad \text { (jet caused by MV) }\end{array}$ & $\begin{array}{l}\text { MV repair } 2 \times, \text { MV replacement } \\
\text { (Melody), MV replacement } \\
\text { (Epic porcine valve) }\end{array}$ & $\begin{array}{r}5(2013,2014, \\
2015,2017)\end{array}$ & LV, septum, papillary muscles \\
\hline 2 & $2 y$ & $\begin{array}{l}\text { HLHS, MS, AR } \\
\text { (jet caused by AV) }\end{array}$ & $\begin{array}{l}\text { PA banding, Norwood I, Glenn, } \\
\text { MV/AV repair }\end{array}$ & $1(2017)$ & $\begin{array}{l}\text { Papillary muscles, post. } \\
\text { LV free wall, septum }\end{array}$ \\
\hline 3 & $12 \mathrm{y}$ & $\begin{array}{l}\text { HLHS, AR, MR/MS } \\
\text { (jet against anterior } \\
\text { mitral leaflet) }\end{array}$ & $\begin{array}{l}\text { PA banding, AV/MV repair, BT } \\
\text { shunt, Glenn, LT Fontan }\end{array}$ & $\begin{array}{r}4(2005,2006 \\
2007,2017)\end{array}$ & $\begin{array}{l}\text { Septum, below aortic valve, } \\
\text { apex, mitral valve annulus, } \\
\text { LA (left pulmonary } \\
\text { vein orifice) }\end{array}$ \\
\hline 4 & $5 \mathrm{mo}$ & $\begin{array}{l}\text { HLHS, critical AS, MS } \\
\text { (2 small jets of inflow) }\end{array}$ & $\begin{array}{l}\text { Fetal balloon dilatation ( } 21 \mathrm{wk}) \text {, } \\
\text { postnatal balloon dilatation } \\
(0 \mathrm{~d}) \text {, Sano I, AV/MV } \\
\text { repair, Glenn }\end{array}$ & $1(2017)$ & $\begin{array}{l}\text { LV free wall, papillary } \\
\text { muscles, apex, LVOT }\end{array}$ \\
\hline 5 & $10 \mathrm{y}$ & HLHS, AR (central jet) & $\begin{array}{l}\text { Postnatal balloon dilatation }(0 \mathrm{~d}) \text {, } \\
\text { Norwood I, BT shunt, AV/MV } \\
\text { repair, AV replacement }\end{array}$ & $2(2010,2017)$ & $\begin{array}{l}\text { Septum, free LV wall, } \\
\text { papillary muscles }\end{array}$ \\
\hline 6 & $9 \mathrm{y}$ & $\begin{array}{l}\text { HLHS, AR } \\
\quad \text { (broad jet), MS }\end{array}$ & $\begin{array}{l}\text { Postnatal balloon dilatation }(0 \mathrm{~d}) \text {, } \\
\text { AV repair } 2 \times, \text { MV repair } 2 \times\end{array}$ & $\begin{array}{l}3(2010, \\
\quad 2013,2017)\end{array}$ & $\begin{array}{l}\text { Posterior LV wall, septum, } \\
\text { apex, sub-aortic region }\end{array}$ \\
\hline 7 & $7 y$ & $\begin{array}{l}\text { HLHS, severe AR } \\
\text { (jet anterior posterior) }\end{array}$ & $\begin{array}{l}\text { Multiple postnatal balloon } \\
\text { dilatations }(0 \mathrm{~d}) \text {, AV repair } 2 \times\end{array}$ & $2(2011,2017)$ & $\begin{array}{l}\text { Subaortic region, LVOT, } \\
\text { septal myectomy }\end{array}$ \\
\hline
\end{tabular}

Data presented include age of patients, surgeries in chronological order, number of EFE resections, and localization of EFE tissue that was obtained for histological analysis. $E F E$, Endocardial fibroelastosis; $H L H S$, hypoplastic left heart syndrome; $A S$, aortic stenosis; $M S$, mitral stenosis; $M V$, mitral valve; $L V$, left ventricle; $A R$, aortic regurgitation; $P A$, pulmonary artery; $A V$, aortic valve; $M R$, mitral regurgitation; $B T$, Blalock-Taussig; $L T$, lateral tunnel; $L A$, left atrium; $L V O T$, left ventricular outflow tract. 
TABLE 2. Detailed information of patients with diagnosis other than hypoplastic left heart syndrome

\begin{tabular}{llll}
\hline Patient & Age & \multicolumn{1}{c}{ Diagnosis } & Localization of EFE resection \\
\hline 1 & $3 \mathrm{y}$ & MS (parachute valve) & LV, supra-mitral membrane \\
2 & $3 \mathrm{y}$ & MS (jet), AS, fetal balloon dilation $(313 / 7 \mathrm{wk})$ & LV, subaortic region \\
3 & $12 \mathrm{y}$ & MS (3 jets) & LV (posterior MV leaflet) \\
4 & $3 \mathrm{mo}$ & Shone's complex, MS (posteriorly and laterally oriented jet), bicuspid AV & LV, papillary septal muscle \\
5 & $3 \mathrm{y}$ & MS (jet posteriorly) & LV, mitral valve muscle \\
6 & $8 \mathrm{y}$ & AR (central jet), postnatal balloon dilation $(1 \mathrm{~d})$ & LV, posterior, septum \\
\hline
\end{tabular}

In these patients, indication for surgical intervention were either MV stenosis or AV regurgitation causing flow disturbances in the left ventricle. However, imaging studies did not describe any endocardial abnormalities in the sense of EFE before surgery. EFE resection was performed following the cardiac surgeon's ad hoc decision intraoperatively. Age of the patients, diagnosis, and localization of EFE resection are presented. $E F E$, Endocardial fibroelastosis; $M S$, mitral stenosis; $L V$, left ventricle; $A S$, aortic stenosis; $M V$, mitral valve; $A V$, aortic valve; $A R$, aortic regurgitation.

and/or insufficiency promote EFE progression independent of the underlying diagnosis of HLHS. Furthermore, we examined age-specific structural changes of EFE tissue potentially affecting diastolic compliance of the ventricle despite extensive resection of EFE tissue in patients with HLHS.

\section{MATERIALS AND METHODS \\ Ethics Statement}

This research project was approved by and conducted in accordance with the standards of the institutional review board of Boston Children's Hospital. Initially only deidentified discarded human tissue was analyzed but following consent prospectively at the time of HLHS/EFE diagnosis,
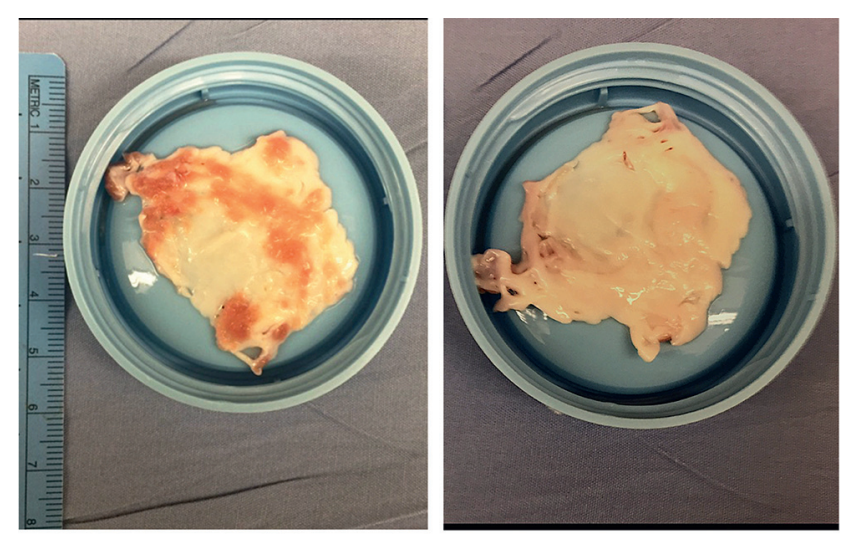

A

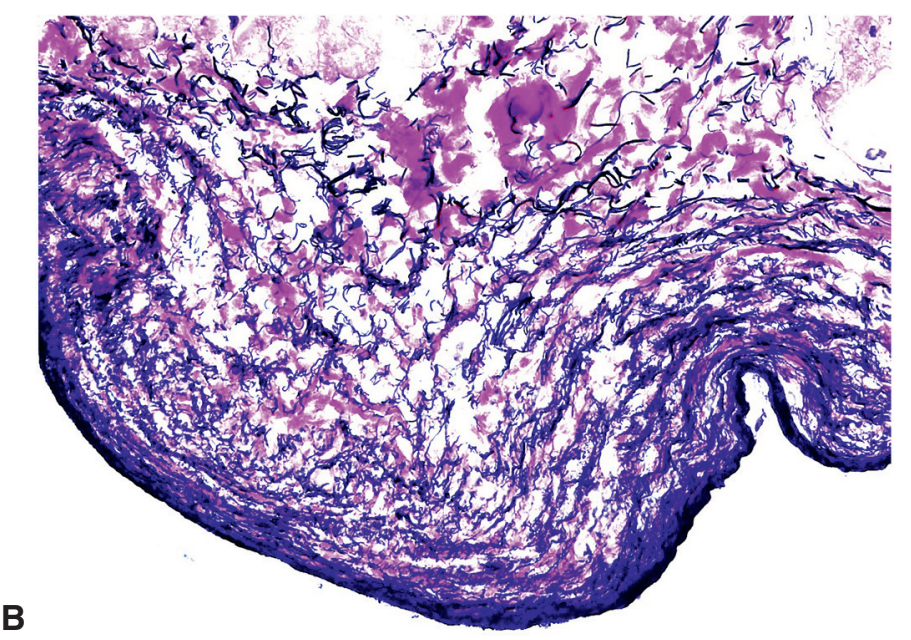

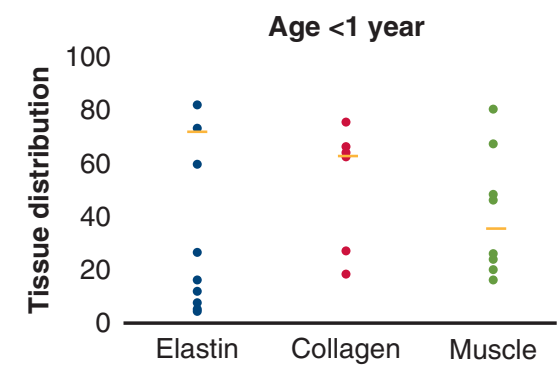
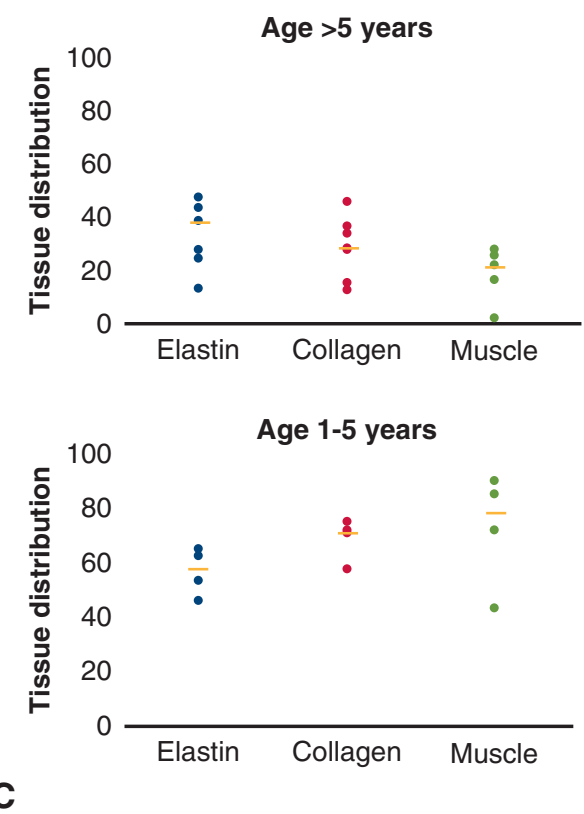

FIGURE 1. A, Macroscopic appearance of resected EFE tissue from the left ventricle of patients with HLHS is shown; left: tissue sample facing the myocardium ( $r e d=$ muscle), right: sample side facing toward the ventricular lumen. B, A representative picture of an Elastin van Giesson stain shows the distribution of collagen (blue) and elastin (black) and muscle (white-yellow) in EFE tissue samples. C, A summary of muscle-collagen-elastin distributions are shown where tissue samples were grouped according to age $(<1$ year, 1-5 years, $>5$ years). There is no significant difference in the distribution within each specific age groups. 


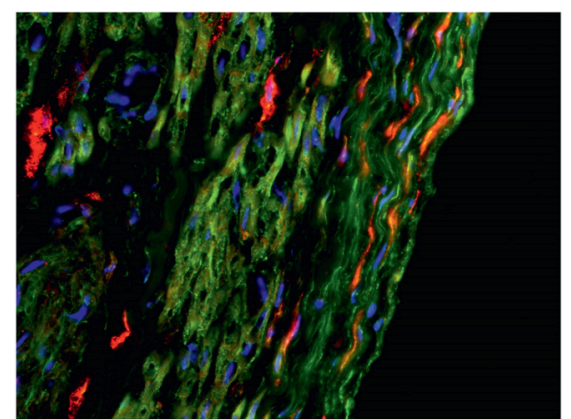

A

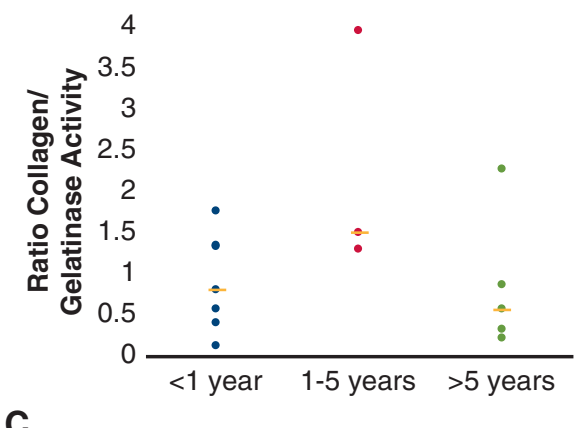

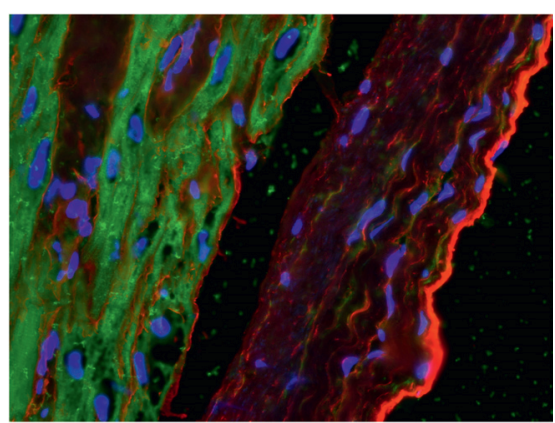

B

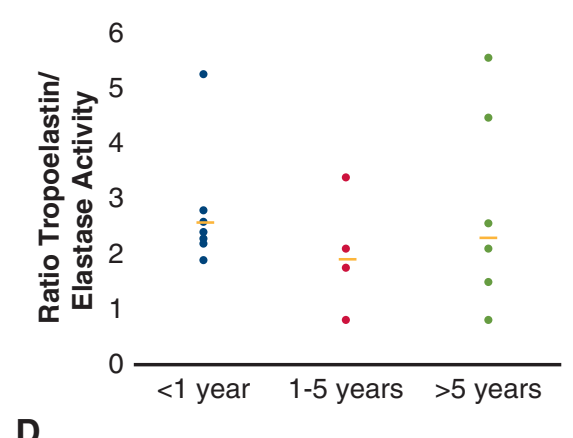

FIGURE 2. A, Representative in situ zymography of gelatinase activity and collagen in EFE tissue obtained from patients diagnosed with hypoplastic left heart syndrome is shown. Gelatinase is shown in green, collagen in red, and nuclei in blue. B, Representative in situ zymography of elastase activity and tropoelastin in EFE tissue obtained from patients diagnosed with hypoplastic left heart syndrome. Elastase is shown in green, tropoelastin in red, and nuclei in blue. C and D, Summary of MMP activity (elastase on the right and gelatinase on the left) per field of vision for different age groups $(<1$ year, 1-5 years, $>5$ years) is shown. There was no significant age-related difference in the balance of MMP activity to collagen/elastin production.

patients' operative details, catheterization details, echocardiographic data, magnetic resonance imaging (MRI) data, and clinical information were obtained and correlated with histologic findings.

\section{Patients}

We analyzed EFE tissue from a total of 24 patients with the diagnosis of HLHS who underwent LV rehabilitation surgery with EFE resection as previously described in more detail at Boston Children's Hospital between December 2011 and March 2018. ${ }^{6}$ Of these 24 patients with HLHS, we received tissue from 17 patients, sent as deidentified discarded tissue, resected during open heart surgeries, and tissue from additional 7 patients, from whom we obtained consent before surgery. In the latter group, we followed the patients, collecting clinical background and imaging data, and obtained EFE tissue from previous resections if available. These patients presented with mitral stenosis and/or aortic stenosis rather than atresia at the time of EFE resection, as detailed in Table 1.

Six patients were included for comparison with diagnoses other than HLHS but with known flow disturbances across aortic and/or mitral valve. In all of these patients, preoperative standard imaging studies (echocardiography and MRI) did not describe EFE. Macroscopic appearance of "EFE-like tissue" in the LV outflow tract diagnosed by the cardiac surgeon performing the surgery led to resection of endocardial tissue in the operating room.

For comparison, we received 2 deidentified discarded tissue samples from the right ventricle from patients without flow disturbances, which was resected in both cases because a right ventriculotomy was performed during the surgical procedure. Furthermore, a healthy rat LV was used as negative control.

All tissue samples, once received from the operating room were immediately embedded in optimal cutting temperature compound, snap frozen, and stored at $-80^{\circ} \mathrm{C}$ for future analysis. Frozen sections were produced from optimal cutting temperature blocks and further processed for microscopic analysis as described in more detail to follow.

\section{Composition of EFE Tissue}

Age-related changes of EFE tissue were examined for the amount of collagen and elastin, vascularity, and cellularity by staining with hematoxylin and eosin, Masson's trichrome (MT), and Van Giessen elastin. All slides were visualized using a Zeiss Axio Observer Z1 fluorescence microscope (Carl Zeiss Microscopy, LLC, White Plains, NY) with a Nikon $20 \times$ objective (numerical aperture $=20 \times / 0.45$; Nikon, Tokyo, Japan). Ten randomly selected fields from each slide were taken and quantification was performed with ImageJ (version 2.0.0-rc-43, obtained from the National Institute of Health, Bethesda, Md). Details about the method of quantifying collagen, elastin, and muscle on histologic sections have been described previously by our group in more detail. ${ }^{1}$

Analysis of tissue remodeling through degradation of elastin fibers and collagen by matrix metalloproteinases was determined by in situ zymography. Unstained frozen slides were either incubated with $40 \mu \mathrm{g} / \mathrm{mL}$ DQ elastin (EnzChek Elastase Assay Kit; Thermo Fisher Scientific, Waltham, Mass) in Dulbecco's Modified Eagle Medium (Gibco; Gaithersburg, Md) and $10 \%$ fetal bovine serum (Gibco) for 1 hour at $37^{\circ} \mathrm{C}$ or in a reaction buffer $\left(0.05 \mathrm{M}\right.$ TRIS- $\mathrm{HCl}, 0.15 \mathrm{M} \mathrm{NaCl}, 5 \mathrm{mM} \mathrm{CaCl}_{2}$, and $0.2 \mathrm{mM} \mathrm{NaN}_{3}$ at $\mathrm{pH}$ 7.6) containing either $40 \mu \mathrm{g} / \mathrm{mL} \mathrm{DQ}$ gelatin or collagen type I or IV (EnzChek Gelatinase/Collagenase Assay Kit) at $37^{\circ} \mathrm{C}$ overnight. Slides, incubated with DQ elastin, were counterstained tropoelastin (1:50; Abcam, Cambridge, Mass) and DAPI (1:1000; Dako, Carpinteria, Calif), and slides incubated with DQ gelatin were counterstained with collagen I (1:50; Novus Biologicals, Littleton, Colo) and DAPI (1:1000; Dako). All chemicals were obtained from Thermo Fisher Scientific. 

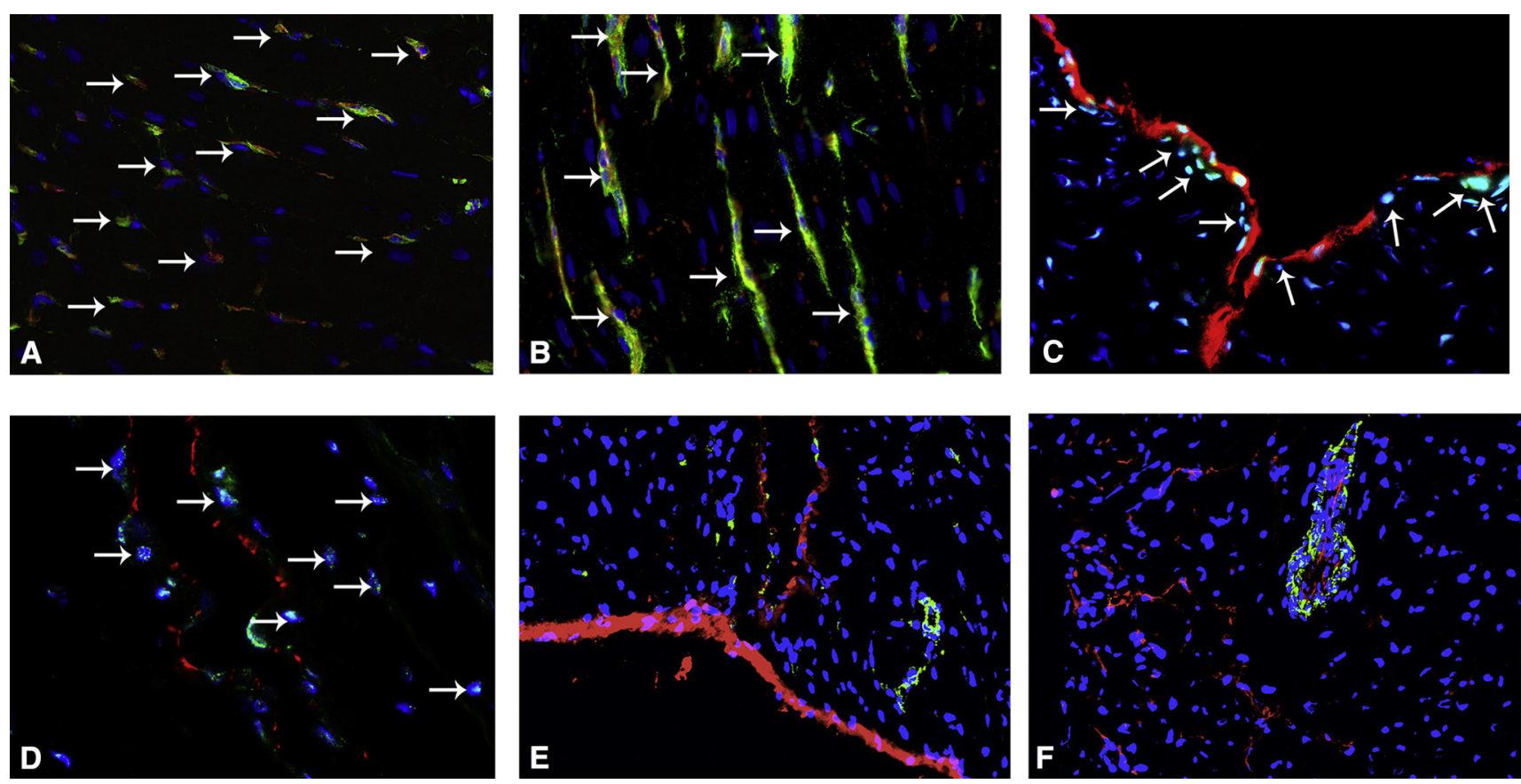

FIGURE 3. Representative sections of EFE and "EFE-like" tissues are displayed. Double-staining of endothelial cells with an endothelial cell marker (CD-31) in red and a fibroblast marker ( $\alpha$-SMA) in green, or endothelial cells with CD-31 (red) and nuclei-positive staining for transcription factors Twist and Slug/Snail (green) is indicative of active EndMT. Nuclei are stained in blue. A, EFE tissue from patients with HLHS is positive for EndMT (CD31/ $\alpha$-SMA double-staining, white arrows); B, "EFE-like" tissue from patients without HLHS is positive for EndMT (CD31/ $\alpha$-SMA double-staining, white arrows); C, EFE tissue and "EFE-like" tissue stained for the transcription factors Twist (white arrows), D, and Slug/Snail (white arrows), respectively, which are indicative of active EndMT when co-localized with endothelial cell nuclei. E, Normal rat heart tissue and F, human right ventricular tissue are negative for EndMT. EFE, Endocardial fibroelastosis; CD31, cluster of differentiation 31; $\alpha$-SMA, $\alpha$-smooth muscle actin; EndMT, endothelial-to-mesenchymal transition; HLHS, hypoplastic left heart syndrome.

\section{Diagnosis of EndMT}

In support of our hypothesis that EndMT is the underlying mechanism for EFE development, the presence of active EndMT was determined in all tissue samples obtained from the operating room. Immunohistochemical double-staining of endothelial cells with an endothelial marker, cluster of differentiation 31 (CD31, 1:100; Dako), and a mesenchymal marker, $\alpha$-smooth muscle actin ( $\alpha$-SMA, 1:100; Abcam) at the same time, is indicative of active EndMT. In addition, all samples were stained for the transcription factors Twist (1:100; Abcam), which regulate EndMT indicated by Twist-positive nuclei. Nuclei were stained for 4',6-diamidino-2-phenylindole (1:1000; Dako). Furthermore, active EndMT was confirmed by staining for the transcription factors Slug/Snail (1:100; Abcam). Co-localization with nuclei in endothelial cells is indicative of active EndMT. As all tissue samples contained some muscle tissue, the myocardium was also analyzed for tissue structure and fibrosis on hematoxylin and eosin and MT as well as immunochistochemical staining for desmin to assess cardiomyocytes and EndMT (1:50; Abcam).

\section{Statistical Analysis}

After confirmation of normal distribution of data, analysis of variance for multiple group comparisons with Bonferroni post-hoc analysis was performed for calculation of statistical significance (STATA, StataCorp, 2015, Stata Statistical Software: Release 14; StataCorp LP, College Station, Tex). Probability values of $\leq .05$ were regarded statistically significant. Data are expressed as mean \pm standard error of the mean.

\section{RESULTS \\ Patients}

The median age of the patients with HLHS at the time of most recent EFE resection was 27 months (interquartile range [IQR] 0-73.5 months) compared with a median age of 36 months (IQR 0.8-73.3 months) in the patients without HLHS $(P=.8)$.

In the patients with HLHS who were consented, apart from the main diagnosis of HLHS, aortic regurgitation, especially following post-natal balloon valvuloplasty, was described in 4 patients; mitral stenosis due to a dysplastic mitral annulus was found in 5 of 7 patients. These valvular diseases caused turbulent flow within the LV in all 7 patients with HLHS who were consented, which has been described throughout their entire medical history. All except 2 patients with HLHS had previous EFE resections. At the time of the most recent resection, all valvar diseases were successfully repaired and no remaining turbulences were described postoperatively and at follow-up neither in echocardiography nor in MRI studies. The median follow-up time after the last EFE resection in the patient with HLHS who was consented was 6.8 months (IQR 4.15-9.45 months) (Table 1). In the patients without HLHS with valvar disease, 


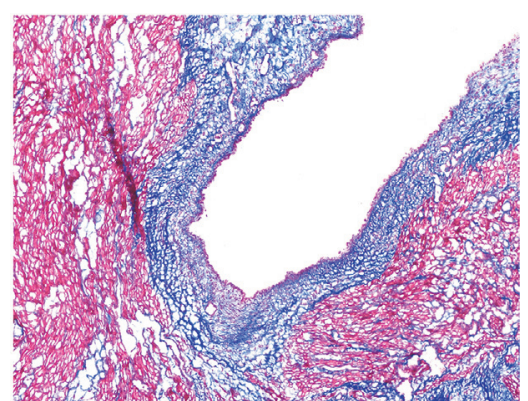

A

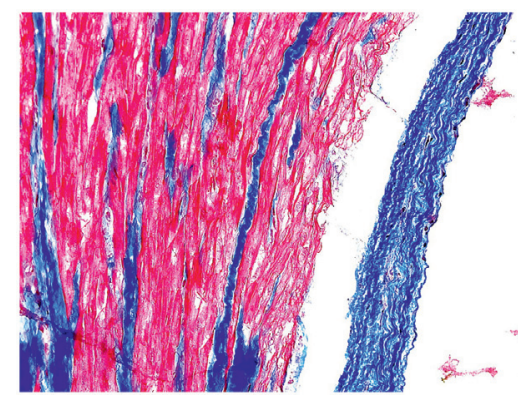

C

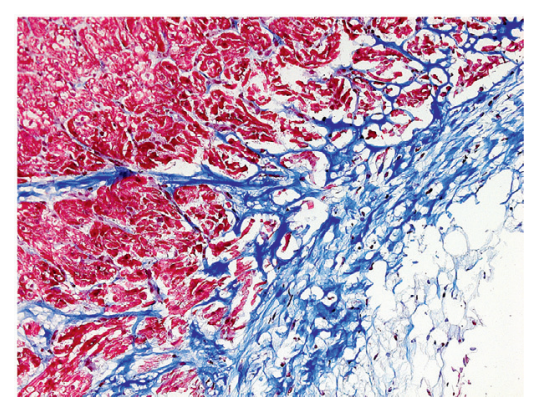

B

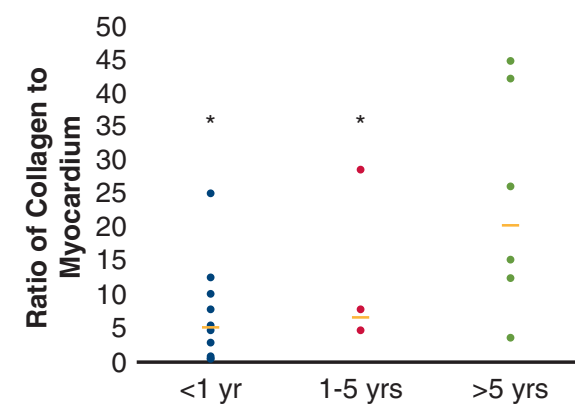

D

FIGURE 4. As indicated by these representative left ventricular tissue samples stained with Masson's trichrome, infiltrative growth patterns of collagen fibers (blue) into the underlying myocardium (red) are associated with increasing age, A, infants ( $<1$ year); B, toddler and preschooler (1-5 years); and C, school-aged children (5-12 years). D, Summary shows significantly greater infiltrative growth in the oldest age group (mean \pm standard error of the mean, ${ }^{*} P<.05$ vs school-aged children).

predominantly a jet across a stenotic mitral valve was present. In 2 cases a regurgitant aortic valve caused a jet toward the left ventricle outflow tract (Table 2).

\section{Composition of EFE Tissue}

The number of nuclei (mean \pm standard error of the mean expressed as nuclei per field of vision) within EFE tissue decreased with increasing age-infants $(<1$ year of age) $282 \pm 63$ versus toddlers and preschoolers (1-5 years of age) $158 \pm 24$ versus school-aged children ( $>5$ years of age with the oldest child at 12 years of age) $94 \pm 12$-but did not reach significance. All tissue remained avascular in all tissue samples. EFE tissue from patients with HLHS consisted predominantly of elastin and collagen fibers with some underlying myocardium. The ratio of muscle to elastin and collagen did not show significant age-related differences within the 3 groups (Figure 1). "EFE-like" tissue obtained from patients without HLHS showed the same characteristics as EFE tissue from patients with HLHS. Collagen and elastin predominated the tissue sections, the number of nuclei decreased with age, and there were no blood vessels found within the tissue.

In situ zymography determined that impaired elastase/ gelatinase activity was not the cause of increased collagen and elastin deposition, and no age-related alteration could be identified (Figure 2). The same characteristics were found in the patients without HLHS with flow disturbances.

\section{Presence of Active EndMT}

Endocardial double-staining with CD31/ $\alpha$-SMA as well as Twist and Slug/Snail-positive stained nuclei, respectively, both indicative of active EndMT, were found not only in all of the 24 samples from patients with HLHS but also in all 6 patients without HLHS. EndMT-positive tissue was not found, however, in right ventricular tissue samples nor in a healthy rat heart (Figure 3).

In all patients with EFE and "EFE-like" tissue, some underlying myocardium was resected (Figure 4). MT stains showed infiltrative growth of collagen bundles from the endocardium into the underlying myocardium of patients with HLHS and patients without HLHS with flow disturbances. The degree of infiltrative growth increases with increasing age in patients with HLHS. In samples of patients $<1$ year of age, the fibrotic tissue is separated from the underlying muscle, whereas samples from older children ( $>2$ years of age), no separation between collagen fibers and myocardium is feasible. The endocardial thickness of fibrotic tissue decreases, whereas infiltrative growth of collagen into the underlying myocardium increases (Figure 5). In contrast, in a healthy rat heart and 

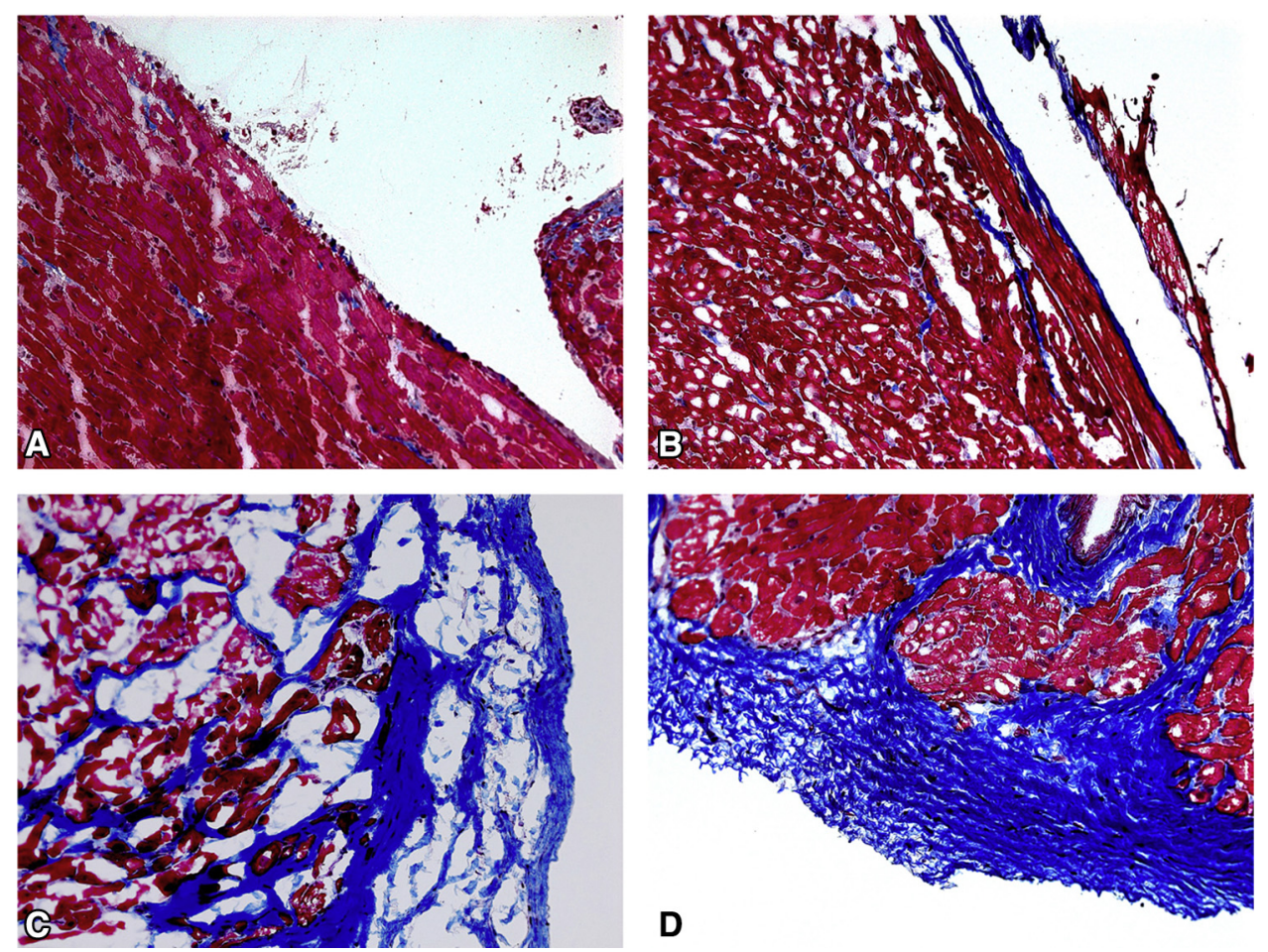

FIGURE 5. Distribution of collagen and muscle tissue on Masson's trichrome stains is shown for A, a normal rat heart, B, normal human tissue, C, LV tissue from a patient with HLHS, and D, LV from a patient without HLHS. Muscle is stained in red and collagen in blue, where of the latter is not limited to the endocardial surface but grows infiltratively into the underlying myocardium in panels C and D.
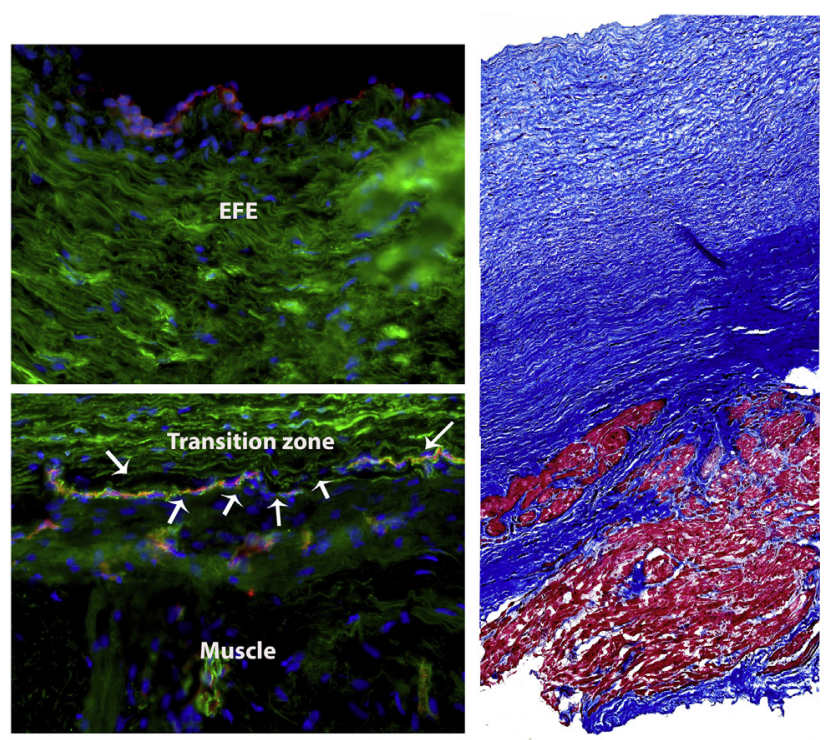

FIGURE 6. EFE tissue that infiltrates the myocardium stains positive for EndMT. A representative section of EFE tissue was stained with Masson's trichrome (blue: collagen fibers, red: myocardium) and correspondingly on the left, the same areas were stained to identify active EndMT (green: fibroblast marker, $\alpha$-SMA; red: endothelial cell marker, CD31; blue: nuclei marker). Double-staining is indicative for EndMT (white arrows), which is more pronounced in the transition zone between endocardial thickening due to EFE tissue and the underlying myocardium. EFE, Endocardial fibroelastosis right ventricle tissue samples, a thin endocardial layer is present that is separated from the underlying myocardium. Localization of endothelial cells within the collagen-rich tissue that infiltrates the myocardium identified multiple double-stained cells for CD31 and $\alpha$-SMA. The double-staining is indicative that intramyocardial fibrotic tissue is also derived through EndMT (Figure 6).

Figure 7 summaries the analytic process from an intraoperative diagnosis of macroscopically appearing EFE tissue, to the unraveling of the mechanistic cause of EFE formation, and the connection to clinical presentation of EFE tissue formation localized to areas of flow disturbances due to valvar defects (see Video 1).

\section{DISCUSSION}

In this study, we established that structural changes of EFE tissue toward a more infiltrative growth into the underlying myocardium and less cellularity occurs with increasing age. Furthermore, we describe for the first time that hemodynamic alterations in the way of jets on the level of the mitral valve or regurgitant flow across an incompetent aortic valve promote localized aggravated EFE growth and reoccurrence despite excessive surgical resection. "EFE-like" tissue growth independent of an underlying HLHS diagnosis shows the same pattern. Following detailed analysis of this "EFE-like" tissue, we determined 


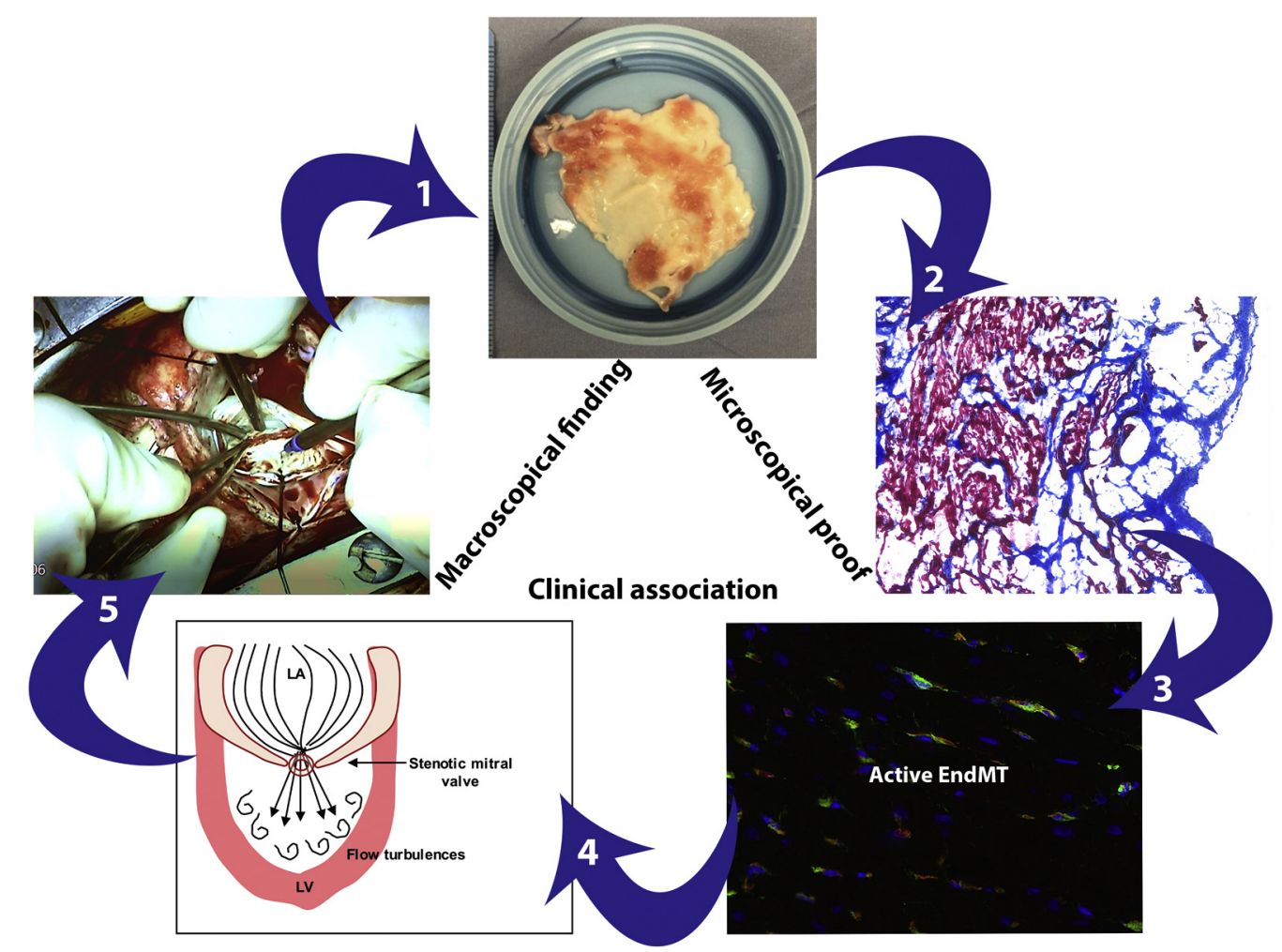

FIGURE 7. The analytic process from intraoperative diagnosis to mechanistic cause connected with clinical presentation is described here. Macroscopically appearing EFE tissue is identified intraoperatively and resected for histological analysis. Immunohistochemical staining establishes EndMT as the underlying mechanism for EFE tissue formation. EFE formation is directly localized to areas of flow disturbances due to valvar defects in the left ventricle. EndMT, Endothelial-to-mesenchymal transition, $L A$, left atrium; $L V$, left ventricle.

that the underlying mechanism for development is EndMT, which we had already reported in detail in patients with EFE associated with HLHS. $^{6}$ Our data further indicate that HLHS alone does not predispose this patient cohort to EFE formation but that the underlying communality is the presence of valvar defects represented as either mitral stenosis or aortic insufficiency. Both valvar defects result in flow disturbances, displayed as jets across the valve onto localized areas of the LV cavity, which responds by endocardial thickening through subendocardial development of EFE tissue. In patients with HLHS, despite several surgical resections of this tissue, progression still occurs unless the valvar defect is repaired.

Another novel finding of this study is that unlike previously suggested, EFE develops and progresses as a distinct layer of fibrotic tissue that can be easily dissected from the underlying myocardium. ${ }^{7}$ Following thorough examination of our resected tissue samples from patients with HLHS and patients without HLHS, all EFE tissue samples contained some degree of muscle tissue. Macroscopically, this finding suggests infiltrative growth of EFE tissue into the underlying myocardium, which we confirm microscopically through MT staining. With increasing age, subendocardial tissue growth takes on a more infiltrative pattern, with collagen and elastin bundles protruding into the adjacent myocardium. Thus, our data show that the main trigger for the development and progression of EFE is flow disturbances, which might also occur in other congenital heart diseases leading to macroscopically "EFE-like" tissue unrelated to the primary diagnosis of HLHS.

In our EFE tissue samples, we could show that localized response of the endocardium to flow disturbances (eg, shear stress) created EFE. It is well known that endothelial cells lining the vasculature respond to alterations in shear stress by changing their shape and function, ${ }^{18}$ since they are exposed to a large variety of biochemical and hemodynamic stimuli. Thus, endothelial cells are required to be highly plastic, which allows for adaptation to alterations in fluid shear forces. Plasticity is essential to maintain homeostasis but pathologic stimuli might alter this process and contribute to disease through adverse plasticity, of which EndMT is an example. ${ }^{19}$ Areas exposed to disturbed blood flow and thus, alterations in shear stress, generate a microenvironment conducive for EndMT. As a consequence, activation of EndMT results in intimal hyperplasia with extracellular matrix remodeling. ${ }^{19,20}$ EFE has the appearance of organized layers resembling 


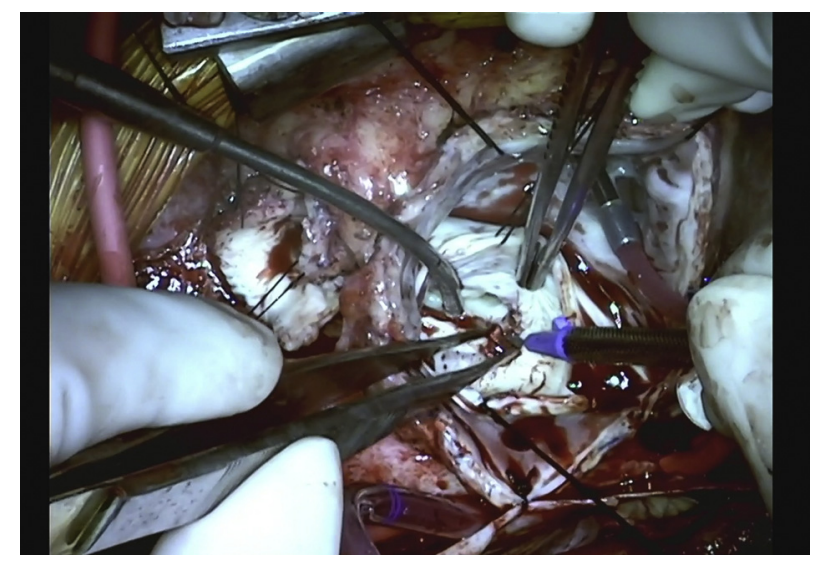

VIDEO 1. This video shows a detailed view of the surgical procedure excising endocardial fibroelastosis from the left ventricular cavity and mitral valve surface. Video available at: https://www.jtcvs.org/article/ S0022-5223(19)31901-4/fulltext.

degenerative intimal hyperplasia of arterial vessel walls. The details on hemodynamic forces and their role in maintaining vascular integrity have been well studied in the vasculature but little is known whether laminar shear stress is protective also for the endocardial endothelial cells. However, it supports the idea that flow disturbances may play a role in endocardial remodeling through EndMT driven by activation of transcription factors that are actively involved in suppression of VE-cadherins and other endothelial-specific proteins. Furthermore, in an animal model of EFE formation that we had previously described in detail, an association with alterations in mechanical forces including flow disturbances across an incompetent aortic valve is associated with formation of EFE. ${ }^{12}$

Functional indicators of EndMT in tissue are increased expression of collagens and elastin, as well as production of enzymes degrading the extracellular matrix, such as matrix metalloproteinase (MMP)-2, MMP-9, or MMP-12 (elastase). The expression of mesenchymal markers corresponds to a newly acquired ability to produce and remodel the extracellular matrix. ${ }^{21}$ With regard to remodeling, activation of MMPs are important following the interruption of cell-cell or cell-matrix contact. In our patient cohort, no defect in the degradative potential of the extracellular matrix of the endocardium or myocardium was found, since all MMPs (MMP12 and MMP2/9) were active in all tissue samples and distribution was focused in areas of active remodeling.

In summary, flow disturbances exposing endocardial endothelial cells to altered shear forces are the main trigger for induction of EndMT leading to reoccurrence of EFE displaying a more infiltrative growth pattern into the underlying myocardium. Supported by our data, we can also conclude that these alterations are not limited to congenital cardiac pathologies associated with HLHS but are related to severe valvar defects with jets onto the endocardial surface appearing to respond by EFE thickening. Targeting EFE therapeutically with surgical resection likely is not sufficient to address reoccurrence and prevent infiltrative growth. A more comprehensive approach by addressing the underlying mechanism of EndMT through localized or systemic therapy is warranted but requires more investigation.

\section{Conflict of Interest Statement}

Authors have nothing to disclose with regard to commercial support.

We thank Breanna Piekarski, BSN, MPH, for contributions regarding institutional review board- and patient-related organization and for collecting tissue samples.

\section{References}

1. Weinberg T, Himmelfarb A. Endocardial fibroelastosis (so-called fetal endocarditis). A report of two cases occuring in siblings. Bull Johns Hopkins Hosp. 1943;26:299.

2. Arya SO, Karpawich PP, Gupta P, Buddhe S, Singh HR, Hussein Y, et al. Primary endocardial fibroelastosis presenting in a young child as incessant ventricular tachycardia and dilated cardiomyopathy. Tex Heart Inst J. 2012;39:714-8.

3. Ni J, Bowles NE, Kim YH, Demmler G, Kearney D, Bricker JT, et al. Viral infection of the myocardium in endocardial fibroelastosis. Molecular evidence for the role of mumps virus as an etiologic agent. Circulation. 1997;95:133-9.

4. Nield LE, Smallhorn JF, Benson LN, Hornberger LK, Silverman ED, Taylor GP, et al. Endocardial fibroelastosis associated with maternal anti-Ro and anti-La antibodies in the absence of atrioventricular block. J Am Coll Cardiol. 2002; 40:796-802.

5. Ursell PC, Neill CA, Anderson RH, Ho SY, Becker AE, Gerlis LM. Endocardial fibroelastosis and hypoplasia of the left ventricle in neonates without significant aortic stenosis. Br Heart J. 1984;51:492-7.

6. Xu X, Friehs I, Hu TZ, Melnychenko I, Tampe B, Alnour F, et al. Endocardial fibroelastosis is caused by aberrant endothelial to mesenchymal transition. Circ Res. 2015;116:857-66.

7. Emani SM, McElhinney DB, Tworetzky W, Myers PO, Schroeder B, Zurakowski D, et al. Staged left ventricular recruitment after single-ventricle palliation in patients with borderline left heart hypoplasia. J Am Coll Cardiol. 2012;60:1966-74.

8. Eisenberg LM, Markwald RR. Molecular regulation of atrioventricular valvuloseptal morphogenesis. Circ Res. 1995;77:1-6.

9. Zeisberg EM, Tarnavski O, Zeisberg M, Dorfman AL, McMullen JR, Gustafsson E, et al. Endothelial-to-mesenchymal transition contributes to cardiac fibrosis. Nat Med. 2007;13:952-61.

10. Johnson F. Anoxia as a cause of endocardial fibroelastosis in infancy. Arch Pathol. 1952;54:237-47.

11. Rieder F, Kessler SP, West GA, Bhilocha S, de la Motte C, Sadler TM, et al Inflammation-induced endothelial-to-mesenchymal transition: a novel mechanism of intestinal fibrosis. Am J Pathol. 2011;179:2660-73.

12. Shimada S, Robles C, Illigens BMW, Casar Berazaluce AM, del Nido PJ Friehs I. Distention of the immature left ventricle triggers development of endocardial fibroelastosis: an animal model of endocardial fibroelastosisIntroducing morphopathological features of evolving fetal hypoplastic left heart syndrome. Biomed Res Int. 2015;2015:1-10.

13. Friehs I, Illigens B, Melnychenko I, Zhong-Hu T, Zeisberg E, del Nido PJ. An animal model of endocardial fibroelastosis. J Surg Res. 2013;182:94-100.

14. Crucean A, Alqahtani A, Barron DJ, Brawn WJ, Richardson RV, O'Sullivan J, et al. Re-evaluation of hypoplastic left heart syndrome from a developmental and morphological perspective. Orphanet J Rare Dis. 2017;12:138.

15. Barry OM, Friedman KG, Bergersen L, Emani S, Moeyersoms A, Tworetzky W, et al. Clinical and hemodynamic results after conversion from single to biventricular circulation after fetal aortic stenosis intervention. Am J Cardiol. 2018;122:511-6. 
16. Weixler V, Hammer PE, Marx GR, Emani SM, del Nido PJ, Friehs I. Flow disturbances and progression of endocardial fibroelastosis-a case report. Cardiovasc Pathol. 2019;42:1-3.

17. Illigens BM, Casar Berazaluce A, Poutias D, Gasser R, del Nido PJ, Friehs I. Vascular endothelial growth factor prevents endothelial-to-mesenchymal transition in hypertrophy. Ann Thorac Surg. 2017;104:932-9.

18. Noria S, Cowan DB, Gotlieb AI, Langille BL. Transient and steady-state effects of shear stress on endothelial cell adherens junctions. Circ Res. 1999;85:504-14. vascular disease and is modulated by fluid shear stress. Cardiovasc Res. 2015; 108:377-86.

20. Yao Y, Jumabay M, Ly A, Radparvar M, Cubberly MR, Bostrom KI. A role for the endothelium in vascular calcification. Circ Res. 2013;113:495-504.

21. Woessner JFJ. Matrix metalloproteinases and their inhibitors in connective tissue remodeling. FASEB J. 1991;5:2145-54.

Key Words: endocardial fibroelastosis, hypoplastic left heart syndrome, endothelial-to-mesenchymal transition 